$\mathrm{Cl}$ i ni cogenetic st udy of mut at i ons in LRRK2 exon 41 in Parkinson's di sease pati ents from 18 countries

\begin{tabular}{|l|l|}
\hline 著者 & Tomi yana H r oyuki \\
\hline $\begin{array}{l}\text { j our nal or } \\
\text { publ i cat i on } \mathrm{t} \text { i t I e }\end{array}$ & Novement Di sor der s \\
\hline vol une & 21 \\
\hline number & 8 \\
\hline page r ange & $1102-1108$ \\
\hline year & $2006-04-18$ \\
\hline URL & ht t p: //hdl . handl e. net $/ 10271 / 83$ \\
\hline
\end{tabular}




\section{Clinicogenetic study of mutations in LRRK2 exon 41 in Parkinson's disease patients from 18 countries}

Character count (Title): 100 characters

5 Hiroyuki Tomiyama, MD, ${ }^{1}$ Yuanzhe Li, MD, ${ }^{1}$ Manabu Funayama, PhD, ${ }^{1,2}$ Kazuko Hasegawa, MD, PhD, ${ }^{3}$ Hiroyo Yoshino, BS, ${ }^{1}$ Shin-ichiro Kubo, MD, PhD, ${ }^{1}$ Kenichi Sato, MD, PhD, ${ }^{1}$ Tatsuya Hattori, MD, ${ }^{4}$ Chin-Song Lu, MD, ${ }^{5}$ Rivka Inzelberg, MD, ${ }^{6}$ Ruth Djaldetti, MD, ${ }^{7}$ Eldad Melamed, MD, ${ }^{7}$ Rim Amouri, PhD, ${ }^{8}$ Neziha Gouider-Khouja, MD, ${ }^{8}$ Faycal Hentati, MD, ${ }^{8}$ Yasuko Hatano, MD, ${ }^{1}$ Mei Wang, MD, PhD, ${ }^{1}$ Yoko Imamichi, ${ }^{1}$ Koichi Mizoguchi, $10 \mathrm{MD},{ }^{9}$ Hiroaki Miyajima, MD, ${ }^{10}$ Fumiya Obata, $\mathrm{PhD},{ }^{11}$ Tatsushi Toda, MD, PhD,,${ }^{12,13}$ Matthew J. Farrer, PhD, ${ }^{14}$ Yoshikuni Mizuno, MD, ${ }^{1}$ and Nobutaka Hattori, $\mathrm{MD}, \mathrm{PhD}^{1,13 *}$

${ }^{1}$ Department of Neurology, Juntendo University School of Medicine, Tokyo, Japan;

${ }^{2}$ Japan Foundation for Aging and Health, Chita, Aichi, Japan;

${ }^{3}$ Department of Neurology, National Hospital Organization, Sagamihara National Hospital, Sagamihara, Japan;

${ }^{4}$ Department of Neurology, Honmachi Neurological Clinic, Nagoya, Japan;

${ }^{5}$ Movement Disorders Unit, Department of Neurology, Chang Gung Memorial Hospital, Taipei, Taiwan;

${ }^{6}$ Department of Neurology, Hillel Yaffe Medical Center, Hadera and Rappaport Faculty of Medicine, Technion, Haifa, Israel;

${ }^{7}$ Department of Neurology, Rabin Medical Center-Beilinson Campus Tel-Aviv University, Sackler School of Medicine, Tel-Aviv, Israel;

${ }^{8}$ Department Head, the National Institute of Neurology of Tunis, Tunis, Tunisia;

${ }^{9}$ Department of Neurology, Shizuoka Institute of Epilepsy and Neurological Disorders, Shizuoka, Japan;

${ }^{10}$ First Department of Medicine, Hamamatsu University School of Medicine, Hamamatsu, Japan;

\footnotetext{
${ }^{11}$ Division of Clinical Immunology, Kitasato University Graduate School of Medical Sciences, Sagamihara, Japan;

${ }^{12}$ Division of Functional Genomics, Osaka University Graduate School of Medicine, Suita, Japan;

${ }^{13}$ CREST, Japan Science and Technology Corporation, Kawaguchi, Saitama, Japan; and ${ }^{14}$ Department of Neuroscience, Mayo Clinic, Jacksonville, FL, USA.
} 
*Correspondence to:

A/Prof. Nobutaka Hattori,

Department of Neurology,

Juntendo University School of Medicine,

2-1-1 Hongo, Bunkyo,

35 Tokyo 113-0033, Japan

Tel.: +81-3-5802-1073

Fax: +81-3-5800-0547

e-mail: nhattori@med.juntendo.ac.jp 
Abstract: We screened LRRK2 mutations in exon 41 in 904 parkin-negative Parkinson's disease (PD) patients (868 probands) from 18 countries across 5 continents. We found 3 heterozygous missense (novel I2012T, G2019S, and I2020T) mutations in LRRK2 exon 41. We identified 11 among 868 PD probands (1.3\%) including 2 sporadic cases and 8 of 130 (6.2\%) autosomal dominant PD families. The LRRK2 mutations in exon 41 exhibited relatively common and worldwide distribution. Among the 3 mutations in exon 41, it has been reported that Caucasian patients with G2019S mutation have a single founder effect. In the present study, Japanese patients with G2019S were unlikely to have a single founder from the Caucasian patients. In contrast, I2020T mutation has a single founder effect in Japanese patients. Clinically, patients with LRRK2 mutations had typical idiopathic PD. Notably, several patients developed dementia and psychosis, and one with I2020T had low cardiac ${ }^{123}$ I-metaiodobenzylguanidine (MIBG) heart/mediastinum ratio, although the ratio was not low in other patients with I2020T or G2019S. Clinical phenotypes including psychosis, dementia, and MIBG ratios are also heterogeneous, similar to neuropathology, in PD associated with LRRK2 mutations.

Key words: genetics; Parkinson's disease; PARK8; leucin rich repeat kinase 2 (LRRK2); cardiac ${ }^{123}$ I-metaiodobenzylguanidine (MIBG) scintigraphy 
Parkinson's disease (PD) is the second most common neurodegenerative disorder with a prevalence of $3 \%$ in individuals older than 65 years of age. ${ }^{1}$ Although the majority of PD cases are sporadic, it is now clear that genetic factors contribute to the pathogenesis of PD. Indeed, alpha-synuclein, ${ }^{2} U C H-L 1,{ }^{3}$ and $L R R K 2^{4,5}$ have been reported as causative genes of autosomal dominant PD (ADPD); and parkin, ${ }^{6} \mathrm{DJ}-1,{ }^{7}$ and $P I N K 1^{8}$ as causative genes of autosomal recessive PD (ARPD). Recently, $L R R K 2$ was identified as the causative gene for $P A R K 8,{ }^{4,5}$ and a common single LRRK2 mutation in exon 41 (G2019S) has been found in mainly Caucasian patients with familial PD. ${ }^{9}$ In the present study, we performed direct sequencing of $L R R K 2$ exon 41 in a large number of patients with familial and sporadic PD of different ethnic origins. We also analyzed the haplotype to determine whether a single haplotype was associated with LRRK2 mutations. In addition, we investigated the clinicogenetic features, including cardiac ${ }^{123}$ I-metaiodobenzylguanidine (MIBG) scintigraphy, of patients with $L R R K 2$ mutations.

\section{Patients and Methods}

All blood samples and clinical information were obtained by the neurologists working in the respective countries after obtaining informed consent from their patients. The study was approved by the ethics review committee of Juntendo University. Diagnosis of PD was made by the participating neurologists based on the presence of parkinsonism, good response to anti-PD treatment, and radiological findings. In all countries, the clinical evaluation was based on the Unified Parkinson's Disease Rating Scale. Controls were evaluated by neurologists to ensure none of them had PD. For control subjects, the mean age of the Japanese was 56.1 (range, 23-98) years and that of the Taiwanese was 34.4 (range, 21-86) years. DNA was prepared using standard methods. The mutation screening was performed as 
described previously. ${ }^{6}{ }^{10}$ Dideoxy cycle sequencing was performed with Big Dye Terminater Chemistry (Applied Biosystems, Foster City, CA). This was followed by exon sequencing on ABI377 and 310 automated DNA sequence analyzers (Applied Biosystems). None of the participants had parkin mutations (including exonic deletions and multiplications), as confirmed by polymerase chain reaction (PCR), direct sequencing, and quantitative assays based on real-time PCR with Taq-Man probes (Applied Biosystems) of all exons. females 437, not reported 54; aged 7-100 years, mean, 47.5 years) including 868 probands from 18 countries (Japan, Korea, Taiwan, China, Philippines, Australia, Israel, Tunisia, Morocco, Turkey, Greece, Poland, Bulgaria, Yugoslavia, United Kingdom, USA, Canada, and Brazil). Asian probands (Japanese, Koreans, Taiwanese, and Chinese) formed the majority (763/868, 87.9\%). The remainder included 72 Caucasian probands (Israelis, Australians, Turkish, Greek, Polish, Bulgarians, Yugoslavs, British, Americans, and Canadians), and 15 North African probands (Tunisians and Moroccans). The other 18 probands were Filipinos ( $n=3$ ), Brazilians $(n=1)$, or could not be specified ( $n=14)$. In this study, families with more than two affected members or two members with a mutation at least in two generations were classified as ADPD, and families with at least two affected siblings in only one generation as (potential or pseudo) ARPD. Among 904 PD patients, we considered 130 families as ADPD, 80 families as ARPD, and 417 patients as sporadic PD. Haplotype analysis was performed as described previously. ${ }^{10,11}$ Genotyping of patients with $L R R K 2$ mutations was performed using the microsatellite markers (D12S2514, D12S2515, D12S2516, D12S2518, D12S2519, D12S2520, D12S2521, D12S2522, and D12S2523) and the SNPs (rs1427263, rs11176013, and rs11564148). ${ }^{10}$ Alleles were sized by GENESCAN (Applied Biosystems). 
Cardiac MIBG scintigraphy was performed with an intravenous injection of $111 \mathrm{MBq}$ of ${ }^{123}$ I-MIBG (Daiichi Radioisotope Laboratories Co., Tokyo, Japan). Early images were 110 obtained 15 minutes and delayed images were obtained 3-4 hours after injection. Whole cardiac MIBG uptake was measured on a planar image as the heart/mediastinum (H/M) activity ratio. 


\section{Results}

\section{Genetic studies}

We found two heterozygous missense mutations (6059T>C; I2020T and 6055G>A; G2019S), which had been reported previously, ${ }^{5,9,12-14}$ and one novel heterozygous missense mutation (6035T>C; I2012T) (Fig. 1a, b). The novel I2012T mutation is also highly conserved across various species, similar to the other previously reported mutation sites of I2020T and G2019S. ${ }^{5,12-14}$ We did not find any of these mutations in 200 chromosomes from normal Taiwanese and 200 chromosomes from normal Japanese populations. We found three Japanese patients (two probands) with the I2020T. Patient A1 with the novel I2012T mutation was of Taiwanese origin.

(6.2\%) ADPD families (one Taiwanese, two Japanese, two Israelis, and three Tunisians) with three different mutations in LRRK2 exon 41. The G2019S mutation was detected in 8 probands (one Japanese, three Israelis, one Moroccan, and three Tunisians). Considering the mode of inheritance, two of 417 sporadic PD patients $(2 / 417=0.5 \%)$ had the G2019S with the age at onset of 41 and 42 years. Including all ethnic groups, five ADPD families (two Israelis and three Tunisians) had the G2019S (5/130 = 3.8\%), and interestingly 1 of 80 potential or pseudo ARPD families had the G2019S $(1 / 80=1.3 \%)$.

Considering each ethnic group, the frequency of PD probands with the G2019S was as follows: $0.1 \%$ (1/763) in Asian people; 4.2\% (3/72) in Caucasian people; and 26.7\% (4/15) in North African people. The frequency of PD probands with the I2020T was 0.3\% (2/763) in Asian people. In addition to the pathogenic mutations, we found a novel single polymorphism (6054C>T) that was synonymous (Y2018Y) in two Korean families. 
The parent and the parent's sibling of Japanese Patients J1 and J2 with the I2020T were born in the Sagamihara area, and the Japanese Patient K1 with the I2020T was also born near the Sagamihara area. The haplotype with five microsatellite markers in this region was exactly the same as that of the Sagamihara family. ${ }^{11,12}$ In contrast, the haplotype of the other Japanese Family B (Patients B1 and B2) with the G2019S was quite different from that of the Caucasian families reported previously (Fig. 2a, b). ${ }^{10}$

\section{Clinical features}

Most patients with LRRK2 mutations in exon 41 showed cardinal signs of idiopathic PD and good responses to levodopa (Table). Other clinical features noted in some patients included dementia, hallucination, delusion, and depression. Patients C1 and C2 with the G2019S mutation developed visual hallucinations, paranoidal thoughts, and dementia. Patient H1 with the G2019S mutation developed hallucinations and delusion. Patients B1 and C2 with the G2019S mutation also suffered from depression. Patient J2 with the I2020T mutation developed severe auditory and visual hallucinations and severe delusion. Psychoses were not induced by medication (levodopa/dopamine agonists) and did not relent when off medication. The doses of anti-PD drugs for Patient J2 were selegiline $2.5 \mathrm{mg} /$ day, cabergoline $3 \mathrm{mg} /$ day, amantadine 150 mg/day, and talipexole 1.2 mg/day. Patient B1 developed upward gaze palsy. None of the patients showed severe autonomic disturbances or amyotrophy. The age at onset of the patients with these three mutations varied widely from 37 to 73 years (average, 55.0 years). In addition to these patients, two grown-up children (aged 36 and 38 years) with mutations were asymptomatic (Family C, Fig. 1b). Pathologic studies could not be conducted in all patients with $L R R K 2$ mutations in this study.

Cardiac MIBG scintigraphy was performed in Patients B1 and B2 with G2019S mutation and Patients J1, J2, and K1 with I2020T mutation (Table). For Patient B1, B2, and 
$\mathrm{K} 1$, the $\mathrm{H} / \mathrm{M}$ ratio was normal. For Patient $\mathrm{J} 1$, the $\mathrm{H} / \mathrm{M}$ ratio was 1.56 in early image and 1.39 in delayed image. For Patient J2, the H/M ratio was 1.45 in early image and 1.30 in delayed image. The cut off value of delayed H/M ratio is 2.09 in Patients B1 and B2, and is 1.45 in Patients J1, J2, and K1 (each cut off value is different because the MIBG scintigraphy was performed in different hospitals). Patient J2 had no ischemic heart disease, chronic heart failure, diabetes mellitus, and no medication history of anti-depressants that may influence MIBG uptake. Notably, Patient J2, who developed severe psychosis, had a low H/M ratio in delayed MIBG images.

\section{Discussion}

The present study revealed that the LRRK2 G2019S mutation spreads worldwide across different ethnic groups with variable frequencies. Whereas the G2019S mutation is quite rare in Asia, this mutation is more frequent around Europe $\mathrm{e}^{9,13,14}$ and especially in North Africa. Demographically, it is possible that the distribution and frequencies of this mutation is associated with human migration history. In a previous report, European and North American patients with the G2019S shared a common ancestral haplotype indicative of a single founder effect. ${ }^{10}$ Indeed, our patients from around Europe and North Africa also had the same haplotype. On the other hand, the haplotype of Japanese Family B was quite different from that of Caucasian patients, suggesting they do not share a common founder and they are of independent origin. The possibility that Japanese patients have a common haplotype on the very narrow region could not be excluded. However, it is unlikely that the Japanese G2019S mutation originated from a common and extremely old founder, because the G2019S mutation is rare in Asian people compared with North African and Caucasian peoples. PARK8 was originally mapped from Japanese Sagamihara family showing late-onset 
ADPD. ${ }^{11}$ The coincidence of haplotype in Japanese Family J, Family K, and Sagamihara family indicates a single founder effect. The same mutation I2020T was found in a Caucasian patient of European origin (personal communication, Dr. Thomas Gasser), ${ }^{5}$ suggesting this mutation also has a worldwide distribution, although the haplotype is unknown at present. Interestingly, G2019S and I2020T mutations affect adjacent codons in the amino acid sequence. In addition to the two mutations, I2012T is also located in the very narrow region within exon 41. This narrow region could be a hot spot and mutations in exon 41 could be relatively frequent. Since $L R R K 2$ consists of 51 exons, it is important to decide which exon(s) of this gene should be firstly screened for mutation analysis. In this regard, worldwide screening of exon 41 prior to all other exons is a reasonable strategy. Analysis of other putatively pathogenic coding substitutions and exon 31 is now also recommended. ${ }^{15}$

With regard to the mode of inheritance, our study showed that not only patients with ADPD but also those with potential ARPD and sporadic PD had LRRK2 mutations. Moreover, we detected the mutations in late- and early-onset PD. A shared LRRK2 G2019S haplotype in the vast majority of carriers argues against de novo occurrence. ${ }^{13}$ Based on the widely variable age at onset and various modes of inheritance, the phenotype of PD patients with LRRK2 mutations must be influenced by stochastic, environmental, and other genetic factors. $^{10}$

The clinical features of most patients with LRRK2 mutations resemble those of patients with typical idiopathic PD. ${ }^{10}$ Previously reported patients with LRRK2 mutations, especially those with G2019S and I2020T, showed no evidence of psychiatric or cognitive dysfunction apart from mild symptoms, despite the long disease duration. 5,11,12,16 However, some of our patients with the LRRK2 mutations developed severe hallucination, moderate dementia, delusion, and depression, even during the early stages of the disease. Both Patients C1 and C2 with G2019S of the same family had psychosis and dementia. On the other hand, 
although of the same family, Patient J2 with I2020T showed psychosis and Patient J1 did not show psychosis. Notably, only Patient J2 developed severe psychosis and had low H/M ratio in delayed MIBG images. In contrast, markedly low H/M ratio was not noted in other patients without psychosis.

MIBG scintigraphy is a clinically useful diagnostic method and has high sensitivity. MIBG uptake shows remarkable decrease in Lewy body positive PD patients from the early stage, except for some of the patients with stage I. Indeed, in a previous study, a more severely reduced H/M ratio was seen in dementia with Lewy bodies compared with PD, and the strong negative correlation between $\mathrm{H} / \mathrm{M}$ ratio and Hoehn-Yahr stage in PD was observed, suggesting that Lewy body pathology itself may cause low MIBG uptake. ${ }^{17}$ Histopathological studies showed a high incidence of Lewy bodies in the sympathetic ganglia and visceral autonomic nervous system, ${ }^{18}$ and the presence of Lewy bodies and Lewy neurites in the cardiac plexus in PD. ${ }^{19}$ These findings may account for low cardiac uptake of MIBG in Lewy body disease, reflecting actual cardiac sympathetic denervation, which precedes the neuronal loss in the sympathetic ganglia. ${ }^{19,20}$ In this regard, the reduced cardiac uptake of MIBG in Patient $\mathrm{J} 2$ is compatible with Lewy body disease, and Patient $\mathrm{J} 2$ with severe psychosis may have cardiac sympathetic denervation and spread of Lewy bodies. On the other hand, Patients B1 and B2 with G2019S and Patients J1 and K1 with I2020T may have no Lewy bodies, similar to most patients of the Sagamihara family. ${ }^{11,12}$ The normal MIBG uptake in autosomal recessive juvenile parkinsonism (AR-JP), which is caused by the parkin gene, ${ }^{21,22}$ generally without Lewy bodies, psychosis, and dementia, ${ }^{23}$ supports our findings concerning MIBG scintigraphy and clinical features.

In the present study, the clinical features and cardiac uptake of MIBG varied even in patients with the same I2020T mutation, and also even in members of the same family. Indeed, one PD patient with the I2020T mutation from the Sagamihara family had Lewy 
bodies although most of the other family members were negative for Lewy bodies (personal communication, Dr. Ogino). Thus, variable pathologies such as positive and negative Lewy body, and variable clinical phenotypes were observed in patients with LRRK2 mutations.

In the previously reported German-Canadian family and Western Nebraska family, the phenotypes of PARK8-linked parkinsonism with signs of dementia, diffuse Lewy body disease (DLBD), progressive supranuclear palsy (PSP), and motor neuron degeneration, showed pathological features of synucleinopathies and tauopathies. ${ }^{24-27}$ In the present study, one patient with G2019S showed upward gaze palsy like PSP although downward gaze palsy was not seen. Interestingly, some of the patients with LRRK2 R1441C mutation had Lewy bodies, while patients with Y1699C mutation in the German-Canadian family and most patients with I2020T of the Sagamihara family had no Lewy bodies. ${ }^{5,24-27}$ Thus, clarification of the function of $L R R K 2$ product and its interaction with other proteins is important for our understanding of the pathogenesis of dopaminergic neuronal death with or without Lewy bodies.

The domain structure of LRRK2 includes five conserved major functional domains including leucine-rich repeat (LRR), a Roc (Ras in complex proteins) domain, a COR domain (C-terminal of Roc), a tyrosine kinase catalytic domain (TyrKc), and a WD40 domain. ${ }^{5}$ The I2012T, G2019S, and I2020T mutations are located in the kinase domain. The G2019S and I2020T mutations may be involved in the alteration of kinase activities of the $L R R K 2$ product. ${ }^{28}$ Furthermore, this protein may be potentially associated with phosphorylation of both alpha-synuclein and tau. ${ }^{4,5,29}$ Indeed, concurrent pathology of alpha-synuclein and tau accumulation within the same aggregates was reported previously. ${ }^{29}$ Therefore, the kinase activity of the $L R R K 2$ product could be a key event in the accumulation and aggregation of these unfolded proteins within the degenerating neurons, ${ }^{4,5}$ and could be an upstream event. 
In our study, we showed that PD caused by LRRK2 mutations is common and widespread, showing variable pathology and variable clinical phenotypes including psychosis and dementia. Our study could have major implications on the clinical understanding of phenotype-genotype correlations and could help in genetic counseling for PD. Insights into the pathogenesis of the disease may lead to new treatment of PD and neurodegenerative disorders.

\section{Acknowledgments}

The authors thank all the participants. DNA samples from abroad were sent by Drs. Sibel Özekmekçi, Bülent Elibol, Hülya Apaydin, Tanya Gurevich, N. Giladi, Amos Korczin, Sharon Hassin, Joseph Jankovic, Tracy Brown, Michael G. Schlossmacher, Mark Gidesblatt, Beom S. Jeon, Myung Sik Lee, Kim Jae Woo, Dione Chua, Vladimir S. Kostic, Roberto Giugliani, Sevasti Bostantzopoulou, and Anna Kowalska. This study was supported in part by the Japanese Ministry of Education, Culture, Sports, Science and Technology; the Japanese Ministry of Health, Labor and Welfare; National Parkinson Foundation; and Uehara Memorial Foundation.

Words count (Text): 2624 words 


\section{References}

1. Lang AE, Lozano AM. Parkinson's disease. First of two parts. N Engl J Med 1998;339:1044-1053.

2. Polymeropoulos MH, Lavedan C, Leroy E, et al. Mutation in the alpha-synuclein gene identified in families with Parkinson's disease. Science 1997;276:2045-2047.

3. Leroy E, Boyer R, Auburger G, et al. The ubiquitin pathway in Parkinson's disease. Nature 1998;395:451-452.

4. Paisán-Ruíz C, Jain S, Evans EW, et al. Cloning of the gene containing mutations that cause PARK8-linked Parkinson's disease. Neuron 2004;44:595-600.

5. Zimprich A, Biskup S, Leitner P, et al. Mutations in LRRK2 cause autosomal-dominant parkinsonism with pleomorphic pathology. Neuron 2004;44:601-607.

6. Kitada T, Asakawa S, Hattori N, et al. Mutations in the parkin gene cause autosomal recessive juvenile parkinsonism. Nature 1998;392:605-608.

7. Bonifati V, Rizzu P, van Baren MJ, et al. Mutations in the DJ-1 gene associated with autosomal recessive early-onset parkinsonism. Science 2003;299:256-259.

8. Valente EM, Abou-Sleiman PM, Caputo V, et al. Hereditary early-onset Parkinson's disease caused by mutations in PINK1. Science 2004;304:1158-1160.

9. Nichols WC, Pankratz N, Hernandez D, et al. Genetic screening for a single common LRRK2 mutation in familial Parkinson's disease. Lancet 2005;365:410-412.

10. Kachergus J, Mata IF, Hulihan M, et al. Identification of a novel LRRK2 mutation linked to autosomal dominant parkinsonism: Evidence of a common founder across European populations. Am J Hum Genet 2005;76:672-680.

11. Funayama M, Hasegawa K, Kowa H, et al. A new locus for Parkinson's disease (PARK8) maps to chromosome 12p11.2-q13.1. Ann Neurol 2002;51:296-301. 
12. Funayama M, Hasegawa K, Ohta E, et al. An LRRK2 mutation as a cause for the parkinsonism in the original PARK8 family. Ann Neurol 2005;57:918-921.

13. Gilks WP, Abou-Sleiman PM, Gandhi S, et al. A common LRRK2 mutation in idiopathic Parkinson's disease. Lancet 2005;365:415-416.

14. Di Fonzo A, Rohe CF, Ferreira J, et al. A frequent LRRK2 gene mutation associated with autosomal dominant Parkinson's disease. Lancet 2005;365:412-415.

15. Mata IF, Kachergus JM, Taylor JP, et al. Lrrk2 pathogenic substitutions in Parkinson's disease. Neurogenetics 2005;17:1-7.

16. Aasly JO, Toft M, Fernandez-Mata I, et al. Clinical features of LRRK2-associated Parkinson's disease in central Norway. Ann Neurol 2005;57:762-765.

17. Nagayama H, Hashimoto M, Ueda M, et al. Reliability of MIBG myocardial scintigraphy in the diagnosis of Parkinson's disease. J Neurol Neurosurg Psychiatry 2005;76:249-251.

18. Wakabayashi K, Takahashi H, Ohama E, et al. Lewy bodies in the visceral autonomic nervous system in Parkinson's disease. In: Narabayashi H, Nagatsu N, Yanagisawa N, Mizuno Y, editors. Adv Neurol. New York: Lippincott-Raven; 1993. p.609-12

19. Iwanaga K, Wakabayashi K, Yoshimoto M, et al. Lewy body-type degeneration in cardiac plexus in Parkinson's and incidental Lewy body diseases. Neurology 1999;52:1269-1271.

20. Orimo S, Amino T, Itoh Y, et al. Cardiac sympathetic denervation precedes neuronal loss in the sympathetic ganglia in Lewy body disease. Acta Neuropathol (Berl) 2005;109:583-588.

21. Suzuki M, Hattori N, Orimo S, et al. Preserved myocardial [123I]metaiodobenzylguanidine uptake in autosomal recessive juvenile parkinsonism: first case report. Mov Disord 2005;20:634-636. 
22. Kitami T, Nomoto T, Nagao T, et al. ${ }^{123}$ I-MIBG myocardial scintigraphy in Juvenile Parkinsonism. Mov Disord 1998;13(suppl 2):247.

23. Lücking CB, Dürr A, Bonifati V, et al. Association between early-onset Parkinson's disease and mutations in the parkin gene. N Engl J Med 2000;342:1560-1567.

24. Wszolek ZK, Vieregge P, Uitti RJ, et al. German-Canadian family (family A) with parkinsonism, amyotrophy, and dementia - Longitudinal observations. Parkinsonism Relat Disord 1997;3:125-139

25. Wszolek ZK, Pfeiffer B, Fulgham JR, et al. Western Nebraska family (family D) with autosomal dominant parkinsonism. Neurology 1995;45:502-505.

26. Zimprich A, Müller-Myhsok B, Farrer M, et al. The PARK8 locus in autosomal dominant parkinsonism: Confirmation of linkage and further delineation of the disease-containing interval. Am J Hum Genet 2004;74:11-19.

27. Wszolek ZK, Pfeiffer RF, Tsuboi Y, et al. Autosomal dominant parkinsonism associated with variable synuclein and tau pathology. Neurology 2004;62:1619-1622.

28. Hernandez DG, Paisán-Ruíz C, Mclnerney-Leo A, et al. Clinical and positron emission tomography of Parkinson's disease caused by LRRK2. Ann Neurol 2005;57:453-456.

29. Duda JE, Giasson BI, Mabon ME, et al. Concurrence of alpha-synuclein and tau brain pathology in the Contursi kindred. Acta Neuropathol (Berl) 2002;104:7-11. 


\section{Legends for figures}

a $\quad \operatorname{I2012T}(6035 \mathrm{~T}>\mathrm{C}) \quad \mathrm{G} 2019 \mathrm{~S}(6055 \mathrm{G}>\mathrm{A}) \quad \mathrm{I} 2020 \mathrm{~T}(6059 \mathrm{~T}>\mathrm{C})$

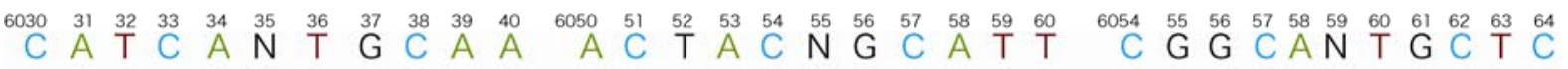
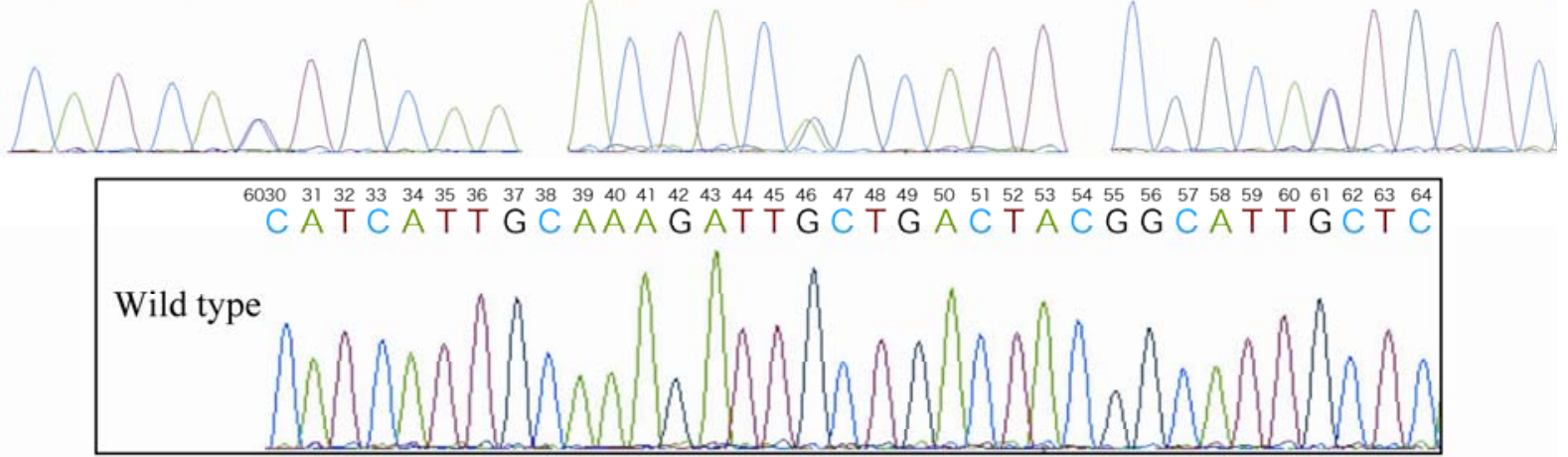

b

Family A

(I2012T, Taiwan)

Family B

(G2019S, Japan)

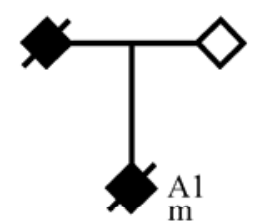

Family G (G2019S, Tunisia)

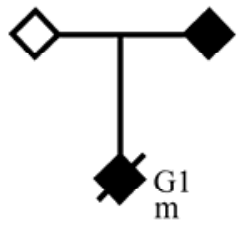

Family H (G2019S, Tunisia)

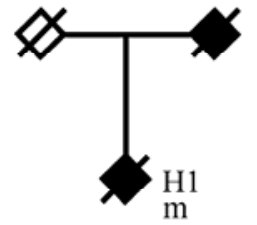

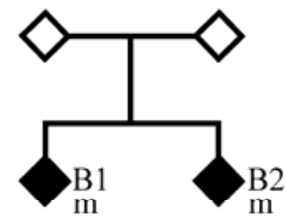

Family C (G2019S, Israel)

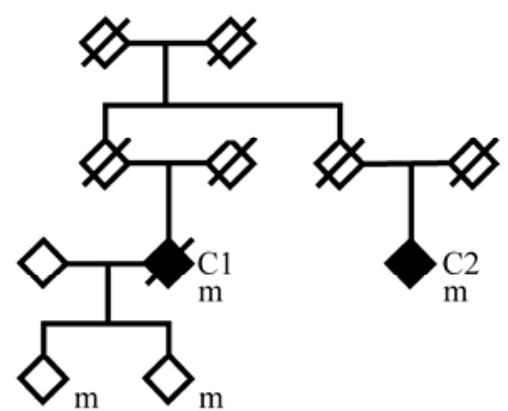

Family I (G2019S, Tunisia)

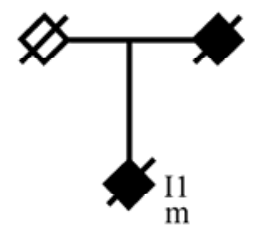

Family D (G2019S, Israel)

Figure 1. (a) Chromatogram showing three mutations in LRRK2 exon 41. A novel 6035T $>$ C (I2012T) mutation was found in Family A. The 6055G>A (G2019S) mutation was found in Families B-I. The 6059T>C (I2020T) mutation was found in Families J and K. (b) Pedigree structures of Families A-D and G-K. Solid symbols denote affected members with PD and symbols of "m" denote members with mutation. To provide confidentiality for participants, some unaffected members and some affected members in whom haplotype analysis was not performed are not shown on the trees. 
a

\begin{tabular}{|c|c|c|c|c|c|c|c|c|c|c|c|c|c|c|c|c|c|c|c|c|c|c|c|c|c|}
\hline \multirow{3}{*}{\multicolumn{2}{|c|}{ Patient }} & \multicolumn{24}{|c|}{ Family (country) } \\
\hline & & \multicolumn{4}{|c|}{ B (Japan) } & \multicolumn{4}{|c|}{ C (Israel) } & \multicolumn{4}{|c|}{$\mathrm{D}$ (Israel) } & \multicolumn{2}{|c|}{ E (Israel) } & \multicolumn{3}{|c|}{ (Morocco } & \multicolumn{2}{|c|}{ G (Tunisia) } & \multicolumn{3}{|c|}{$\mathrm{H}$ (Tunisia) } & \multicolumn{2}{|c|}{ I (Tunisia) } \\
\hline & & \multicolumn{2}{|c|}{ B1 } & \multicolumn{2}{|c|}{ B2 } & \multicolumn{2}{|c|}{$\mathrm{Cl}$} & \multicolumn{2}{|c|}{$\mathrm{C} 2$} & \multicolumn{2}{|c|}{ D1 } & \multicolumn{2}{|c|}{ D2 } & \multicolumn{2}{|c|}{\begin{tabular}{|l|l} 
& E1 \\
\end{tabular}} & & $\mathrm{F} 1$ & & \multicolumn{2}{|c|}{ G1 } & \multicolumn{3}{|c|}{$\mathrm{H1}$} & \multicolumn{2}{|c|}{ I1 } \\
\hline Marker & D12S345 & 210 & $214 i$ & 210 & 232 & 214 & 2141 & 214 & 2101 & 214 & 218 & 214 & 212 & 214 & 231 & 212 & 22 & & 211 & 217 & 214 & & 312 & 226 & 234 \\
\hline & 514 & 295 & 295 ! & 295 & 295 & 291 & 291 i & 291 & $291 !$ & 291 & 295 & 291 & 295 & 291 & 291 & 291 & 29 & & 293 & 285 & 291 & 29 & & 291 & 291 \\
\hline & D12S2515 & $\begin{array}{l}216 \\
216\end{array}$ & $228 !$ & 216 & 228 & 224 & $224 !$ & 224 & $216 !$ & $\begin{array}{l}224 \\
224\end{array}$ & 216 & 224 & 224 & 224 & 216 & 224 & 22 & & 228 & 228 & 224 & 21 & $2: 2$ & 228 & 232 \\
\hline (LRRK2 IVS 30$)$ & D12S2516 & 252 & 252 & 252 & 252 & 254 & 254 & 254 & 252 & 254 & 252 & 254 & 252 & 254 & 254 & 254 & 25 & & 254 & 254 & 254 & 25 & $4: 2$ & 254 & 254 \\
\hline (LRRK2 Exon 3 & rs1427263 & $\mathrm{C}$ & C i & $\mathrm{C}$ & C & A & A i & A & C : & A & $\mathrm{C}$ & A & C & A & A & 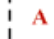 & A & $i$ & A & C & A & & & A & A \\
\hline (LRRK2 Exon 34) & rs11176013 & A & A & A & A & $G$ & G I & $\mathrm{G}$ & A & G & A & G & A & G & G & G & G & 1 & G & A & G & G & 1 & G & G \\
\hline (LRRK2 Exon 34) & rs11564148 & T & $\mathrm{T}$ & $\mathrm{T}$ & $\mathrm{T}$ & A & A i & $\mathrm{A}$ & $\mathrm{T}:$ & A & $\mathrm{T}$ & A & $\mathrm{T}$ & i & A & i & $\mathrm{T}$ & i & $\mathbf{A}$ & $\mathrm{T}$ & A & & $i$ & A & A \\
\hline (LRRK2 IVS 45) & D12S2518 & 154 & 168 & 154 & 168 & 154 & $154 !$ & 154 & $168 !$ & 154 & 154 & 154 & 154 & 154 & 154 & 154 & 15 & & 154 & 168 & 154 & & $4: 1$ & 154 & 154 \\
\hline & D12S2519 & 140 & 1401 & 140 & 132 & 132 & $132 i$ & 132 & $132 i$ & 132 & 132 & 132 & 138 & 132 & 132 & 132 & 14 & & 132 & 138 & 132 & 13 & 21 & 132 & 132 \\
\hline & 520 & $\begin{array}{l}257 \\
257\end{array}$ & $257 i$ & 257 & 254 & 260 & $254 !$ & 260 & $257 !$ & 260 & 260 & 260 & 260 & 260 & 257 & 260 & 25 & $7: 2$ & 260 & 248 & 260 & 25 & $7: 2$ & 260 & 257 \\
\hline & D12S2521 & | 320 & $339 !$ & 320 & 364 & 359 & $363 !$ & 359 & $357 !$ & $\begin{array}{l}359 \\
359\end{array}$ & 367 & 359 & 323 & 359 & 359 & 359 & 32 & & 359 & 324 & \begin{tabular}{l|l}
359 \\
359
\end{tabular} & & $3: 3$ & 359 & 363 \\
\hline & $2 \mathrm{~S} 2522$ & 285 & $281 !$ & 285 & 297 & 297 & $297 !$ & 297 & $297 !$ & 297 & 297 & 297 & 297 & 297 & 297 & 297 & 28 & & 297 & 281 & 297 & 29 & $7 ! 2$ & 297 & 297 \\
\hline & & I 314 & $317 i$ & 314 & 320 & 320 & 3201 & 320 & 3201 & 320 & 320 & 320 & 320 & 320 & 32 & I 320 & 31 & & 320 & 318 & 320 & & 013 & 320 & 320 \\
\hline & D12S1653 & $\begin{array}{l}202 \\
202\end{array}$ & $206 !$ & 202 & 206 & 210 & 218 i & 210 & $206 !$ & $\begin{array}{l}210 \\
210\end{array}$ & 220 & 210 & 220 & 210 & 220 & 210 & 21 & $5: 2$ & 217 & 206 & 217 & & $7: 2$ & 217 & 219 \\
\hline
\end{tabular}

\begin{tabular}{|c|c|c|c|c|c|c|}
\hline \multirow{3}{*}{ Patient } & \multicolumn{6}{|c|}{ Family (country) } \\
\hline & \multicolumn{3}{|c|}{$\mathrm{J}$ (Japan) } & \multicolumn{3}{|c|}{ I'K (Japan)'Sagamihara } \\
\hline & 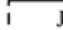 & \begin{tabular}{l|l}
$\mathrm{J} 1$ & $\mathrm{I}$ \\
$\mathrm{J}$
\end{tabular} & $\mathrm{J} 2$ & $\mathrm{I}$ & $\mathrm{K} 1$ & I (Japan) \\
\hline Marker i D12S1631 & 185 & 181,185 & 5177 & 185 & 177 & 185 \\
\hline D12S87 & 155 & $155: 155$ & 157 & 155 & 155 & 155 \\
\hline $\mathrm{D} 12 \mathrm{~S} 345$ & 224 & $214: 224$ & 212 & 224 & 230 & 224 \\
\hline D12S1653 & 222 & 2101222 & 210 & 222 & 216 & 222 \\
\hline $\begin{array}{l}\text { D12S85 } \\
\end{array}$ & 121 & $123: 121$ & 103 & 121 & 103 & 121 \\
\hline
\end{tabular}

Figure 2. Haplotype analysis (a) The haplotype of Families C-I with the G2019S from around

Europe was similar to the previously reported Caucasian family. ${ }^{10}$ Genotypes shared are

highlighted in red. The haplotype of Japanese Family B with the G2019S was different from that of Families C-I and the previously reported Caucasian family. ${ }^{10}$ The size of these markers are corrected using CEPH 1331-01 and -02 as controls. (b) The haplotype of

Families J and K with the I2020T was similar to that of the Japanese Sagamihara family.

Shared genotypes are highlighted in blue. The haplotype with five microsatellite markers in this region spans $10.4 \mathrm{cM}$. 